\title{
Efecto de la castración química en el rendimiento y calidad de carcasa del cuy (Cavia porcellus)
}

\section{Effect of chemical castration on the performance and carcass quality of the guinea pig (Cavia porcellus)}

\author{
Litman Keler Santillán Mendoza', William Bardales Escalante², Luis Homero Zagazeta Llanca ${ }^{3}$
}

\section{RESUMEN}

En la presente investigación se tuvo como objetivo determinar el efecto de la castración química en la ganancia de peso, calidad de carcasa y sabor de cuyes machos (Cavia porcellus). Se utilizaron 30 cuyes de la raza Perú, de 35 días de edad, distribuidos en dos tratamientos (T1: control y T2: castrados con tintura de yodo al $2 \%$ ) y quince repeticiones. La castración se realizó a los 35 días de edad por inyección intratesticular de tintura de yodo al $2 \%$ en dosis de $0.1 \mathrm{ml}$ por testículo, los pesos de los cuyes fueron evaluados hasta los 95 días de edad. No se encontró diferencia estadística significativa en la variable ganancia de peso total ( $p>0.05)$, en el rendimiento de carcasa se encontró diferencia estadística significativa $(\mathrm{p}<0.05)$, siendo mayor en los castrados con pesos promedio de 672.67 gr y en los no castrados un peso promedio de $605.00 \mathrm{gr}$, la evaluación organoléptica sensorial de la carcasa del cuy, se encontraron diferencias estadísticas significativas $(\mathrm{p}<0.05)$ en los aspectos de olor, sabor y aceptabilidad mas no en color y textura, siendo favorable para los cuyes castrados. Con relación a la utilidad se determinó que el T 1 (testigo) reporta un ingreso libre de S/. 2.57 por Cuy vendida siendo mayor en los Castrado ( $\mathrm{T}$ 2) S/. 5.03, ya que esta presenta una mejor calidad de carcasa sin lesiones en la región dorso posterior de cada animal. En conclusión, el rendimiento de carcasa, los aspectos organolépticos y la relación beneficio - costo; hacen que se considere el T2 como la mejor alternativa de producción..

Palabras clave: Castración química, Cavia porcellus

\begin{abstract}
The objective of this investigation was to determine the effect of chemical castration on weight gain, carcass quality and taste of male guinea pigs (Cavia porcellus). 30 guinea pigs of the Perú breed, 35 days old, were distributed in two treatments (T1: control and T2: castrated with $2 \%$ iodine tincture) and fifteen repetitions. Castration was performed at 35 days of age by intratesticular injection of $2 \%$ iodine tincture at a dose of $0.1 \mathrm{ml}$ per testicle, the weights of the guinea pigs were evaluated until 95 days of age. No significant statistical difference was found in the variable total weight gain ( $p>0.05)$, in the carcass yield significant statistical difference was found ( $p$ $<0.05$ ), being higher in the castrates with average weights of $672.67 \mathrm{gr}$ and in those not castrated an average weight of $605.00 \mathrm{gr}$, the sensory organoleptic evaluation of the guinea pig's carcass, significant statistical differences (p $<0.05$ ) were found in the aspects of smell, taste and acceptability but not in color and texture, being favorable for castrated guinea pigs. Regarding utility, it was determined that $\mathrm{T} 1$ (witness) reports a free income of S /. 2.57 for pig sold being older in the castrated (T 2) S/. 5.03, since it presents a better quality of carcass without lesions in the back posterior region of each animal. In conclusion, the carcass performance, the organoleptic aspects and the benefit - cost ratio; They make T2 be considered the best production alternative.
\end{abstract}

Keywords: Chemical castration, Cavia porcellus

${ }^{1}$ Egresado de la Universidad Nacional Toribio Rodríguez de Mendoza de Amazonas.

${ }^{2}$ Universidad Nacional Toribio Rodríguez de Mendoza de Amazonas.

${ }^{3}$ Zootecnista 


\section{INTRODUCCIÓN}

La producción de cuyes en Perú es en general una actividad rural la cual está localizada en mayor énfasis en la zona de la Sierra, en donde predomina el sistema de crianza tradicional - familiar. Es ahí donde la producción pecuaria familiar juega un rol importante para dar solución al problema del hambre en la región y también puede y genera una parte importante de los alimentos necesarios para el mercado interno del país, mejorando así la seguridad alimentaria y nutricional, y por ende contribuyendo significativamente al desarrollo nacional (Espíritu y Herrera, 2011).

En la crianza de los nuevos genotipos de cuyes existe la necesidad de intensificar y mejorar la eficiencia de las prácticas de producción y post-producción de una manera sostenible (Espíritu y Herrera, 2011), es por ello que resulta importante observar hacia el mercado una vez concluida la etapa de crianza (Chauca, 1997). Procesos como la castración se emplea frecuentemente con el fin de alterar el desarrollo sexual de los machos induciendo cambios regresivos de comportamiento y estructurales, se trata de un procedimiento estándar en la producción animal para modificar el comportamiento agresivo de los machos y eliminar características indeseables de su carne en algunas especies (Hernández, 2002).

Según Caycedo, 2000, citado por Shiroma et al., 2004, la castración química consiste en la aplicación de sustancias esclerosantes a nivel intratesticular que tienen como objeto atrofiar el parénquima causando la esterilidad del macho.

Tabla 01. Sustancia y dosis utilizadas para castrar químicamente.

\begin{tabular}{|c|c|}
\hline Sustancia química & Dosis \\
\hline \multicolumn{2}{|l|}{ Alcohol yodado } \\
\hline $2 \%$ & $0,5 \mathrm{ml} /$ testículo \\
\hline $\begin{array}{c}\text { Alcohol yodado } \\
5 \%\end{array}$ & $0,1 \mathrm{ml} /$ testículo \\
\hline \multicolumn{2}{|l|}{ Tintura de yodo 2} \\
\hline$\%$ & $0.1 \mathrm{ml} /$ testículo \\
\hline \multicolumn{2}{|l|}{ Ácido láctico } \\
\hline $10 \%$ & $1,2 \mathrm{ml} /$ testículo \\
\hline \multicolumn{2}{|c|}{ Fuente. Shiroma., et al 2004.} \\
\hline \multicolumn{2}{|c|}{$\begin{array}{l}\text { Una investigación realizada en la Facultad de } \\
\text { Ciencias Agropecuarias de la Universidad Nacional } \\
\text { de Trujillo - Perú por Agurto Julio, se analizó los } \\
\text { efectos de la castración química con alcohol yodado } \\
\text { vs ácido láctico sobre la disminución de la } \\
\text { agresividad sexual, ganancia de peso y rendimiento } \\
\text { de la canal, en la que se llegó a la conclusión de que la } \\
\text { castración con alcohol yodado consiguió mejorar los }\end{array}$} \\
\hline
\end{tabular}

parámetros zootécnicos establecidos en la investigación y progresar en el comportamiento agresivo entre animales, comparando con la técnica de castración con ácido láctico y el grupo testigo (Agurto, 2014).

\section{MATERIAL Y MÉTODO}

Se construyó 2 pozas con dimensiones de 1.50 x 1.00 x $0.45 \mathrm{~m} 3$ (largo x ancho x profundidad), con su respectiva identificación por cada unidad experimental.

La población estuvo conformada por 30 cuyes machos elegidos aleatoriamente con características homogéneas de edad (35 días), con peso promedio de 577.3 gr. Cada unidad experimental alojó a 15 animales con su respectiva identificación, provistos de un comedero tipo tolva de plástico de $2 \mathrm{~kg}$ de concentrado y un bebedero de arcilla, de $500 \mathrm{ml}$ de capacidad.

La castración, se realizó a los 35 días de edad teniendo en cuenta los siguientes pasos:

Paso 1. Un asistente sujeto con una mano e inmediatamente se fijó los testículos entre los dedos de su otra mano.

Paso 2. Se realizó la desinfección y la persona responsable inyectó $0.1 \mathrm{ml} /$ testículo de la tintura de yodo al $2 \%$ por vía intratesticular con una aguja y jeringa de tuberculina.

\section{Análisis estadístico}

Los resultados obtenidos de la castración fueron analizados mediante análisis de varianza - ANOVA, con el uso dol programa SPSS, con un nivel de sig cificancia ( ) del $5 \%$ y un nivel de confianza (1- ) del $95 \%$.

\section{RESULTADOS}

\section{Índices Productivos \\ - Ganancia de peso.}


Tabla 02. Análisis de varianza ANOVA de los pesos iniciales

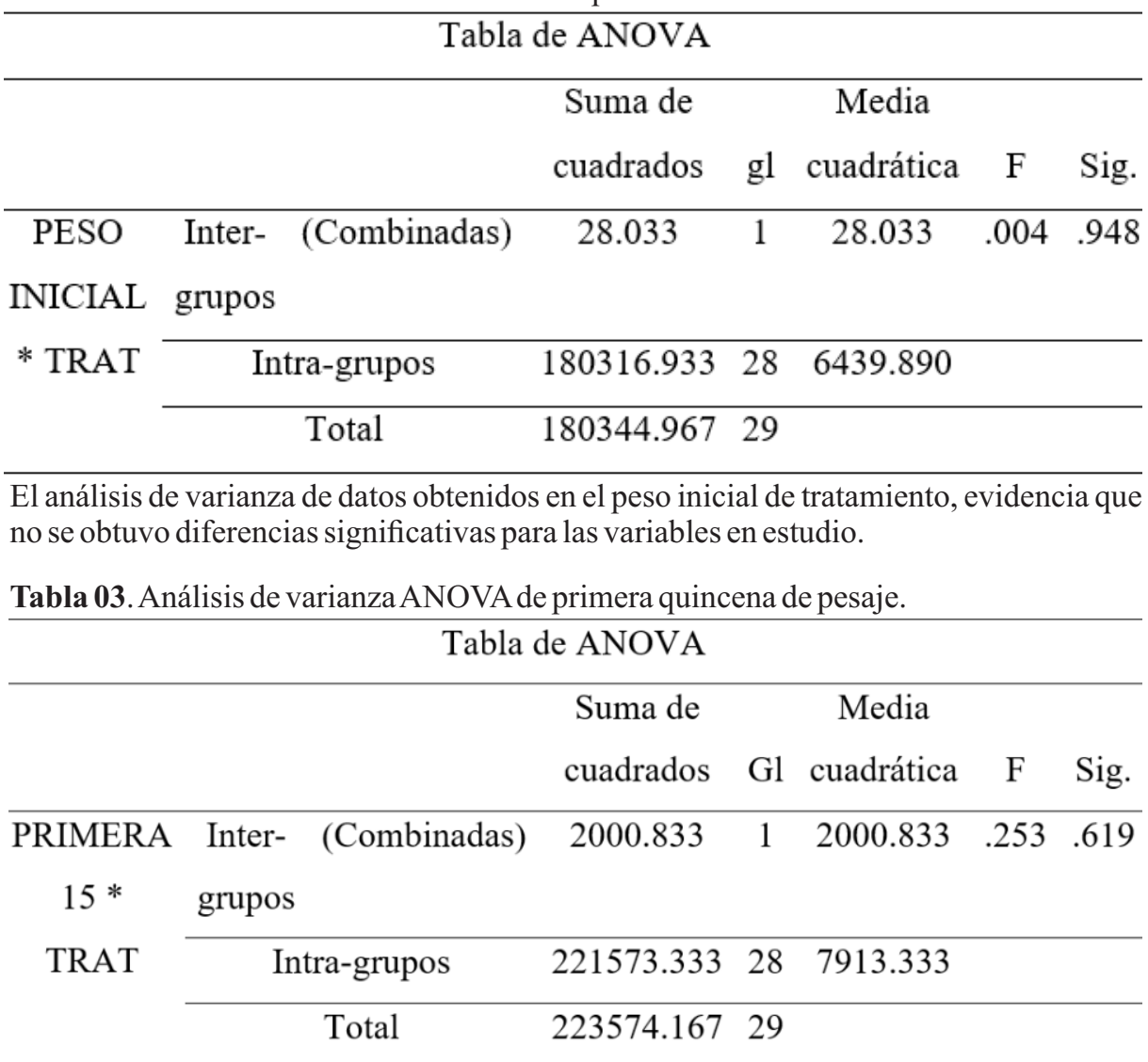

Valores obtenidos del control de peso de la primera quincena.

El análisis de varianza de datos obtenidos, evidencia que no se obtuvo diferencias significativas para las variables en estudio

Tabla 04. Análisis de varianza ANOVA de segunda quincena de pesaje.

\begin{tabular}{|c|c|c|c|c|c|c|c|}
\hline \multicolumn{8}{|c|}{ Tabla de ANOVA } \\
\hline & & & Suma de & & Media & & \\
\hline & & & cuadrados & $\mathrm{gl}$ & cuadrática & $\mathrm{F}$ & Sig. \\
\hline \multirow{4}{*}{$\begin{array}{l}\text { SEGUNDA } \\
15 * \text { TRAT }\end{array}$} & Inter- & (Combinadas) & 6900.833 & 1 & 6900.833 & .853 & .364 \\
\hline & grupos & & & & & & \\
\hline & \multicolumn{2}{|c|}{ Intra-grupos } & 226516.667 & 28 & 8089.881 & & \\
\hline & \multicolumn{2}{|r|}{ Total } & 233417.500 & 29 & & & \\
\hline
\end{tabular}

Valores obtenidos del control de peso de la primera quincena.

El análisis de varianza de datos obtenidos, evidencia que no se obtuvo diferencias significativas para las variables en estudio

Tabla 05. Análisis de varianza ANOVA de tercera quincena de pesaje.

\begin{tabular}{|c|c|c|c|c|}
\hline $\begin{array}{l}\text { TERCERA } \\
15 *\end{array}$ & $\begin{array}{l}\text { Inter- (Combinadas) } \\
\text { grupos }\end{array}$ & 13440.833 & 1 & $13440.833 \quad 1.577$ \\
\hline \multirow[t]{2}{*}{ TRAT } & Intra-grupos & 238633.333 & 28 & 8522.619 \\
\hline & Total & 252074.167 & 29 & \\
\hline
\end{tabular}

Valores obtenidos del control de peso de la tercera quincena.

El análisis de varianza de datos obtenidos, evidencia que no se obtuvo diferencias significativas para las variables en estudio 
Tabla 06. Análisis de varianza ANOVA de la cuarta quincena de pesaje.

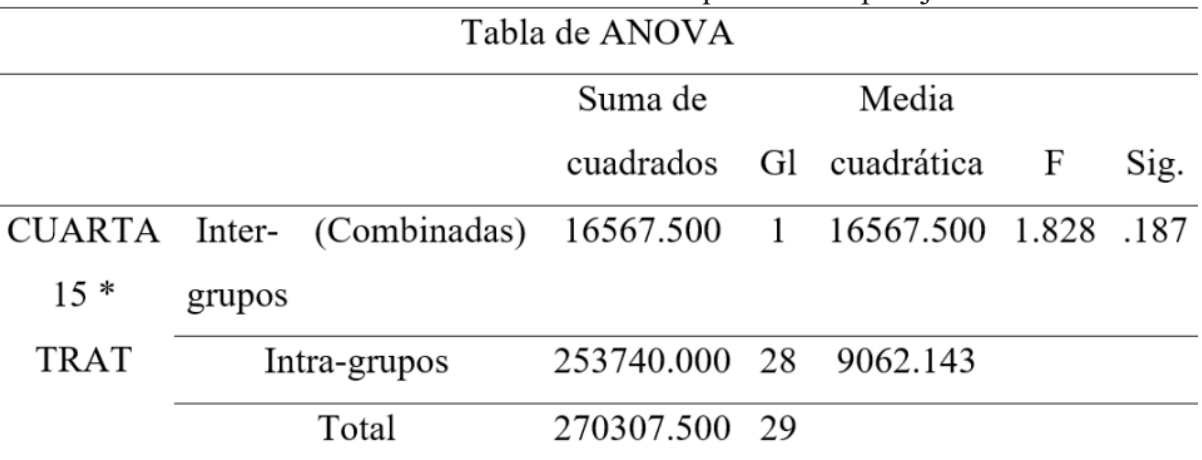

Valores obtenidos del control de peso de la primera quincena.

El análisis de varianza de datos obtenidos, evidencia que no se obtuvo diferencias significativas para las variables en estudio

- Rendimiento de carcasa.

Tabla 07. Análisis de varianza ANOVA del rendimiento de carcasa.

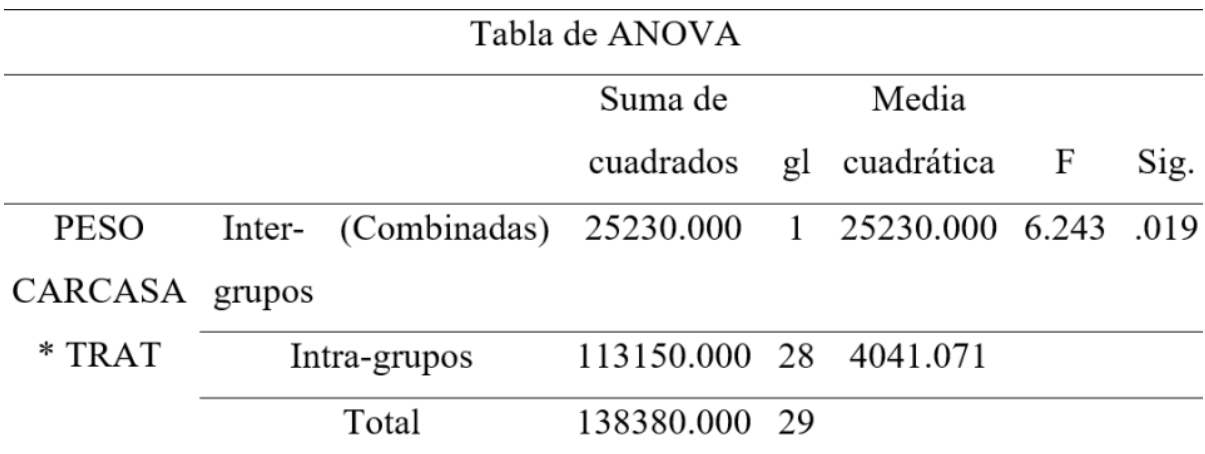

Valores obtenidos del sacrificio de cada animal.

De los valores obtenidos del análisis de varianza se obtuvo como resultado que existe diferencia significativa en el rendimiento de carcasa $(p<0.5)$, entre los tratamientos

- Calidad organoléptica.

Tabla 08. Análisis de varianza ANOVA de las pruebas organolépticas realizadas a cada tratamiento.

\begin{tabular}{|c|c|c|c|c|c|c|}
\hline \multicolumn{7}{|c|}{ ANOVA de un factor } \\
\hline & & $\begin{array}{l}\text { Suma de } \\
\text { cuadrados }\end{array}$ & Gl & $\begin{array}{c}\text { Media } \\
\text { cuadrática }\end{array}$ & $\mathrm{F}$ & Sig. \\
\hline \multirow[t]{3}{*}{ COLOR } & $\begin{array}{l}\text { Inter- } \\
\text { grupos }\end{array}$ & .450 & 1 & .450 & 3.857 & .065 \\
\hline & $\begin{array}{l}\text { Intra- } \\
\text { grupos }\end{array}$ & 2.100 & 18 & .117 & & \\
\hline & Total & 2.550 & 19 & & & \\
\hline \multirow[t]{3}{*}{ OLOR } & $\begin{array}{l}\text { Inter- } \\
\text { grupos }\end{array}$ & 8.450 & 1 & 8.450 & 72.429 & .000 \\
\hline & $\begin{array}{l}\text { Intra- } \\
\text { grupos }\end{array}$ & 2.100 & 18 & .117 & & \\
\hline & Total & 10.550 & 19 & & & \\
\hline SABOR & $\begin{array}{l}\text { Inter- } \\
\text { grupos }\end{array}$ & 8.450 & 1 & 8.450 & 33.800 & .000 \\
\hline
\end{tabular}




\begin{tabular}{ccccccc} 
& $\begin{array}{c}\text { Intra- } \\
\text { grupos }\end{array}$ & 4.500 & 18 & .250 & & \\
\cline { 2 - 7 } & Total & 12.950 & 19 & & & \\
\hline TEXTURA & $\begin{array}{c}\text { Inter- } \\
\text { grupos }\end{array}$ & .450 & 1 & .450 & 3.857 & .065 \\
\cline { 2 - 7 } & $\begin{array}{c}\text { Intra- } \\
\text { grupos }\end{array}$ & 2.100 & 18 & .117 & & \\
\cline { 2 - 7 } & Total & 2.550 & 19 & & & \\
\hline ACEPTABILIDAD & $\begin{array}{c}\text { Inter- } \\
\text { grupos }\end{array}$ & 5.000 & 1 & 5.000 & 18.750 & .000 \\
\cline { 2 - 7 } & $\begin{array}{c}\text { Intra- } \\
\text { grupos }\end{array}$ & 4.800 & 18 & .267 & & \\
\cline { 2 - 7 } & Total & 9.800 & 19 & & & \\
\hline
\end{tabular}

Valores obtenidos de las pruebas organolépticas realizadas.

De los valores obtenidos del análisis de varianza se obtuvo como resultado que existe diferencia significativa en las pruebas organolépticas $(\mathrm{p}<0.5)$ para el olor, sabor $y$ aceptabilidad; la textura y el color no mostraron significancia entre los tratamientos $(\mathrm{p}>0.05)$.

\section{DISCUSIÓN}

El peso total en los cuyes del tratamiento 2 (castración química con tintura de yodo al $2 \%$ ) $(983.7$ gr) fue mayor, pero sin significancia estadística $(\mathrm{P}>0,05)$ en comparación con los cuyes del tratamiento 1 (Testigo) (938.0 gr), lo cual concuerdan con los estudios realizados por Ladera, (2009) quien al comparar 3 tratamientos: T1 (Testigo) (836,4 gr), T2 (Extirpación de espículas) (860,5 gr) y T3 (Castración Química) (725,5 gr), como resultado obtuvo que T1 y T2 presentaron diferencias estadísticas significativas ante $\mathrm{T} 3$ debido a que este último obtuvo un menor ganancia de peso, este resultado puede estar relacionado al estrés provocado en las primeras semanas por la aplicación química de tintura de yodo al $2 \%$ en los testículos.

Si comparamos la ganancia de peso total del $\mathrm{T} 2$ (408. 27 gr) frente al T 1 (360.67 gr) no se encontró diferencia estadística significativa $(\mathrm{P}>0,05)$ para ganancia de peso total, a diferencia de lo reportado por Pujada y Astori, (2012) en Perú, quienes evaluaron 24 cuyes castrados con tintura de yodo al 2 $\%$ con una dosis de $0.1 \mathrm{ml}$ intratesticular, de 35 días de edad, durante 9 semanas frente a un grupo testigo; los resultados reflejaron diferencias significativas $(p<0.05)$ entre los dos grupos para el peso total, lo cual podría estar influenciado a que los animales de estudio están en ambientes diferentes y con una alimentaciones distintas.

Por otra parte Vega et al., (2012), en una investigación donde utilizaron 24 cuyes cruzados Perú-Inti, de 25 a 35 días de edad y distribuidos en dos grupos: castrados con tintura de yodo $2 \%$ $(0.1 \mathrm{~mL} /$ testículo $)$ y no castrados, afirman que la ganancia de peso fue mayor en los animales castrados $(\mathrm{p}<0.01)$, con pesos al beneficio de $(837.9$ gr castrados vs 738.4 gr enteros) y ganancias de peso diaria de (9.06 gr castrados vs 6.94 gr enteros), resultados que son inferiores a esta investigación donde se obtuvo pesos al beneficio de (938.0 gr no castrados vs 983.67 gr cuyes castrados), en la cual se obtuvo que la ganancia de peso en los cuyes castrados fue superior a los cuyes no castrados, en términos de diferencia numérica mas no estadística.

Los resultados obtenidos en la investigación en cuanto al rendimiento a la canal con relación al peso vivo obtenido luego del ayuno de 12 horas pre sacrificio, indican que $\mathrm{T} 2$ es el tratamiento superior con diferencia significativa $(\mathrm{P}<0.05)$ alcanzando un $63.6 \%$ a $72.9 \%$ en comparación con T1 que muestran valores de $50.5 \%$ a $69.5 \%$ de rendimiento respectivamente. Estos son similares a los valores obtenidos por Caycedo, et al., (2011), Chauca, (1997) y Apráez, et al., (2011) quienes reportan rendimientos a la canal de 65 a $69 \%, 64,37 \%$ y 65 a $68 \%$ respectivamente, siendo los dos últimos valores con 24 horas de ayuno.

En la presente investigación se encontró que la castración química mejoró las propiedades organolépticas de: olor, sabor, aceptabilidad, lo cual concuerda con lo mencionado por Apráez et al., (2010) quien señala que la castración química mejora las propiedades organolépticas de la carne y la calidad de la canal para su comercialización. 


\section{CONCLUSIONES}

La castración química con tintura de yodo al $2 \%$ genera mayor ganancia de pesos al beneficio en términos numéricos mas no estadísticos $(\mathrm{p}>0.05)$, mejorando el rendimiento de carcasa en castrados (68.4\%) en comparación a los no castrados (64.5\%) con diferencia estadística significativa de los animales castrados ante los enteros.

En relación al aspecto organoléptico de la carcasa del cuy se obtuvo diferencia significativa del T 2 con respecto al $\mathrm{T} 1$, encontrando que los cuyes castrados tienen un mejor olor, sabor y aceptabilidad.

En relación al beneficio - costo, el T1 reportó S/.2.53 por cuy vendido mientras que el T2 reportó S/. 5.03 siendo esta la mayor utilidad en comparación al grupo control.

\section{REFERENCIAS BIBLIOGRÁFICAS}

Agurto, J. 2014. Efecto de la castración química con alcohol yodado y con ácido láctico sobre la disminución de la agresividad sexual, ganancia de peso y rendimiento de carcasa en (Caviaporcellus).

Caycedo, A., Zamora, A., Echeverry, S., Enríquez, R., Ortega, E., Burgos, M., Caycedo., 2011. Producción Sostenible de Cuyes. Pasto (Colombia): Centro de PublicacionesUniversidad de Nariño: 2011; pp: 92-103. ISBN: 978-958-8609-003.

Chauca, L., 1997. Producción de cuyes (Cavia porcellus). Roma: Organización de las Naciones Unidas para la Agricultura y la Alimentación, 1997; pp: 37-49, 59-61. ISBN 92-5-304033-5.

Hernández, A., \& Fernández, L., 2002. Castración: Una alternativa que facilita el manejo de los cuyes en ceba. Asociación Cubana de Producción Animal ACPA, 20. Recuperado el Enero de 2015, de Asociación Cubana de Producción Animal.

Pujada, H., Vega, J., y Astocuri, K. 2012. Efectos de la castración química en el comportamiento productivo y conductual del cuy. $\mathrm{R}$ e c u p e r a d o d e : http://dx.doi.org/10.15381/rivep.v2311.881

Shiroma P., 2004. Efecto de la castración química con alcohol yodado sobre el crecimiento y rendimiento de la canal en cuyes (Cavia porcellus). Tesis de Médico Veterinario. Lima: Universidad Alas Peruanas. 41 p.

Ladera, A., 2009. Control Hormonal de la Reproducción. En L. Aliaga, R. Moncayo, E. Rico, \& A. Caycedo, Producción de Cuyes (Primera ed., pág. 808). Lima, Perú: Fondo Editorial UCSS. Recuperado el 12 de Marzo de 2016.

Apráez, J., Fernández, L., \& Hernandez, A., 2010. Efecto del sexo y de la castración en el comportamiento productivo y la calidad de la canal en cuyes. Vet. Zootec, 25.

Espíritu, R., y Herrera, E., 2011. Crianza de cuyes manejo tecnificado. Jauja, Perú: CEDAL. Consultado: 2 de agosto del 2013. $\mathrm{R}$ e c u p e r a d o d e : http://cedalperu.org/wpcontent/uploads/201 3/02/modulo3_crianzacuy_manejotecnificado. pdfCruz, H. (2008).

Vega, J., 2012. Efecto de la castración química en el comportamiento productivo y conductual del cuy. RIVER, pág. 2. 